Virginia Commonwealth University VCU Scholars Compass

\title{
Symptom Domain Groups of the Patient-Reported Outcomes Measurement Information System Tools Independently Predict Hospitalizations and Re-hospitalizations in Cirrhosis
}

Kavish R. Patidar

Virginia Commonwealth University

Leroy R. Thacker

Virginia Commonwealth University

James B. Wade

Virginia Commonwealth University

See next page for additional authors

Follow this and additional works at: http://scholarscompass.vcu.edu/psych_pubs

Part of the Psychiatry and Psychology Commons

(C) Springer Science+Business Media New York 2017

Downloaded from

http://scholarscompass.vcu.edu/psych_pubs/71

This Article is brought to you for free and open access by the Dept. of Psychiatry at VCU Scholars Compass. It has been accepted for inclusion in Psychiatry Publications by an authorized administrator of VCU Scholars Compass. For more information, please contact libcompass@vcu.edu. 


\section{Authors}

Kavish R. Patidar, Leroy R. Thacker, James B. Wade, Melanie B. White, Edith A. Gavis, Andrew Fagan, Richard K. Sterling, Michael Fuchs, Mohammad S. Siddiqui, Scott Matherly, Richard T. Stravitz, Arun J. Sanyal, Puneet Puri, Velimir A. Luketic, and Jasmohan S. Bajaj 


\title{
Symptom Domain Groups of the Patient-Reported Outcomes Measurement Information System Tools Independently Predict Hospitalizations and Re-hospitalizations in Cirrhosis
}

\author{
Kavish R. Patidar ${ }^{1}$ - Leroy R. Thacker ${ }^{3}$ James B. Wade ${ }^{2}$ Melanie B. White ${ }^{1}$. \\ Edith A. Gavis ${ }^{1} \cdot$ Andrew Fagan $^{1} \cdot$ Richard K. Sterling ${ }^{1} \cdot$ Michael Fuchs $^{1}$ • \\ Mohammad S. Siddiqui $^{1} \cdot$ Scott Matherly $^{1} \cdot$ Richard T. Stravitz $^{1} \cdot$ Arun J. Sanyal $^{1}$ • \\ Puneet Puri $^{1} \cdot$ Velimir A. Luketic ${ }^{1} \cdot$ Jasmohan S. Bajaj $^{1}$
}

Received: 26 December 2016/ Accepted: 21 February 2017/Published online: 3 March 2017

(C) Springer Science+Business Media New York 2017

\begin{abstract}
Background Patient-Reported Outcomes Measurement Information System (PROMIS) tools can identify healthrelated quality of life (HRQOL) domains that could differentially affect disease progression. Cirrhotics are highly prone to hospitalizations and re-hospitalizations, but the current clinical prognostic models may be insufficient, and thus studying the contribution of individual HRQOL domains could improve prognostication.

Aim Analyze the impact of individual HRQOL PROMIS domains in predicting time to all non-elective hospitalizations and re-hospitalizations in cirrhosis.

Methods Outpatient cirrhotics were administered PROMIS computerized tools. The first non-elective hospitalization and subsequent re-hospitalizations after enrollment were recorded. Individual PROMIS domains significantly contributing toward these outcomes were generated using principal com-
\end{abstract}

Electronic supplementary material The online version of this article (doi:10.1007/s10620-017-4509-y) contains supplementary material, which is available to authorized users.

Jasmohan S. Bajaj

jsbajaj@vcu.edu

1 Division of Gastroenterology, Hepatology and Nutrition, Virginia Commonwealth University and McGuire VA Medical Center, 1201 Broad Rock Boulevard, Richmond, VA, USA

2 Department of Psychiatry, Virginia Commonwealth University and McGuire VA Medical Center, Richmond, VA, USA

3 Department of Biostatistics, Virginia Commonwealth University and McGuire VA Medical Center, Richmond, VA, USA ponent analysis. Factor analysis revealed three major PROMIS domain groups: daily function (fatigue, physical function, social roles/activities and sleep issues), mood (anxiety, anger, and depression), and pain (pain behavior/impact) accounted for $77 \%$ of the variability. Cox proportional hazards regression modeling was used for these groups to evaluate time to first hospitalization and re-hospitalization.

Results A total of 286 patients [57 years, MELD 13, 67\% men, $40 \%$ hepatic encephalopathy (HE)] were enrolled. Patients were followed at 6-month (mth) intervals for a median of $38 \mathrm{mths}$ (IQR 22-47), during which $31 \%$ were hospitalized [median IQR mths 12.5 (3-27)] and 12\% were rehospitalized [10.5 mths (3-28)]. Time to first hospitalization was predicted by HE, HR 1.5 (CI 1.01-2.5, $p=0.04$ ) and daily function PROMIS group HR 1.4 (CI 1.1-1.8, $p=0.01$ ), independently. In contrast, the pain PROMIS group were predictive of the time to re-hospitalization HR 1.6 (CI 1.1-2.3, $p=0.03$ ) as was HE, HR 2.1 (CI 1.1-4.3, $p=0.03$ ).

Conclusions Daily function and pain HRQOL domain groups using PROMIS tools independently predict hospitalizations and re-hospitalizations in cirrhotic patients.

Keywords Cirrhosis - Healthcare-related quality of life · Patient-reported outcomes - Hepatic encephalopathy · Patient-Reported Outcomes Measurement Information System

$\begin{array}{ll}\text { Abbreviations } \\ \text { HE } & \begin{array}{l}\text { Hepatic encephalopathy } \\ \text { Model for End-Stage Liver Disease Health- } \\ \text { MELD }\end{array} \\ \text { Related Quality of Life (HRQOL) } \\ \text { PRO } & \begin{array}{l}\text { Patient-reported outcomes } \\ \text { Patient-Reported Outcomes Measurement } \\ \text { Information System }\end{array} \\ \text { CAT } & \begin{array}{l}\text { Computerized adaptive measurement system }\end{array}\end{array}$


$\begin{array}{ll}\text { IRQ } & \text { Interquartile range } \\ \text { HR } & \text { Hazards ratio }\end{array}$

CI Confidence interval

\section{Introduction}

Cirrhosis is the 12th leading cause of overall death in the USA [1]. Its prevalence will likely continue to increase due to the epidemic of nonalcoholic fatty liver disease [2]. Cirrhosis is also a major cause of healthcare expenditure [3], most of which is due to hospitalizations and re-hospitalizations [4]. Furthermore, cirrhosis-related re-hospitalizations continue to remain excessively high with rates ranging between 36 and 53\% [5, 6] and likely attributable to hepatic encephalopathy (HE). Current methods to predict hospitalizations (and re-hospitalizations) remain challenging as using objective measures such as the Model for End-Stage Liver Disease (MELD), serum sodium, number of medications on discharge, age, history of other complications of cirrhosis, and gender (i.e., male sex), may be inadequate [7-12]. In addition, with the progression of cirrhosis, there is also a parallel impairment in patient-reported outcomes (PROs) such as health-related quality of life (HRQOL) [13-15]. An impaired HRQOL in cirrhosis can predict mortality $[13,16]$ and could contribute to the significant financial and social burden to patients and their families $[13,17]$. Recent data suggest that even with prior HE, cirrhotic patients have good insight regarding their HRQOL [18], and these PROs can add a subjective component to the prediction of outcomes such as HE development [19].

Currently, the majority of research on health domains within HRQOL measures has been studied within a framework of an overall "total score" [20-23]. This approach is not helpful to define individual PRO domains that could predict negative outcomes [24-26]. Therefore, focusing on specific domains or domain groups that specifically result in hospitalizations/re-hospitalizations could guide clinicians toward improving those in preventing these outcomes.

The Patient-Reported Outcomes Measurement Information System computerized adaptive testing (PROMIS CAT) [27-29] is a valid and dynamic instrument to measure and monitor changes of HRQOL in cirrhosis [30, 31]. It has several individual domains that can potentially serve as predictors for hospitalizations/re-hospitalizations in addition to objective parameters in cirrhosis. Therefore, the aim of this study was to prospectively analyze the additive impact of individual HRQOL domains generated using PROMIS tools compared to objective markers of disease severity in predicting time to all hospitalizations and rehospitalizations in patients with cirrhosis.

\section{Materials and Methods}

\section{Study Population}

Patients with cirrhosis were enrolled prospectively from the hepatology clinics at Virginia Commonwealth University Medical Center and McGuire VA Medical Center from November 2009 to December 2015. Patients who were excluded were those who were not able to give informed consent and did not understand English. Because of the potential impact on different domains within HRQOL, we purposefully excluded those who were on disability before diagnoses of cirrhosis, with other significant end-stage organ diseases (i.e., congestive heart failure, chronic obstructive pulmonary disease requiring oxygen, and renal disease requiring dialysis) [32], and current cancer diagnoses [33]. Similarly, we also excluded patients with non-HE psychiatric conditions requiring hospitalizations, on chronic antipsychotics, and on anti-seizure medications [34]. All included patients had cirrhosis proven on a clinical basis involving laboratory tests, imaging findings, endoscopic findings, and liver biopsy if available.

Demographic data and medication use were collected on all patients. The etiology of cirrhosis was categorized into viral hepatitis $\mathrm{C}$, viral hepatitis $\mathrm{C}$ and alcoholic, alcoholic, nonalcoholic steatohepatitis, and other (auto-immune hepatitis, etc.). The severity of liver disease at enrollment was assessed in all patients by the MELD score. Serum ammonia $(\mu \mathrm{mol} / \mathrm{L})$, serum sodium $(\mathrm{mmol} / \mathrm{L})$, and serum albumin $(\mathrm{g} / \mathrm{dL})$ were recorded at time of enrollment. Education was recorded in years. Prior history of episodes of HE were determined by history (patient and caregiver), chart review, and if a patient was on treatment for HE. In addition, history of other cirrhosis complications (ascites and variceal hemorrhage) was recorded at enrollment. The study protocol was approved by the McGuire VA and Virginia Commonwealth University institutional review boards.

\section{PROMIS CAT Tool}

The PROMIS CAT tools assess the following 11 domains: (1) anger; (2) anxiety; (3) depression; (4) fatigue; (5) pain behavior (behavior the patients perform as a result of the pain); (6) pain interference (how does pain interfere with their daily activities); (7) physical function;(8) satisfaction with discretionary social activities (spending time in recreation and friends, etc.); (9) satisfaction with social roles (ability to perform roles expected toward family members, work and potential dependents); (10) sleep disturbance (how does impaired sleep impact their daily activities); and (11) sleep-related impairment (how is the 
sleep itself impaired). Each item of the PROMIS tools was developed individually using patients' representative responses of the 2000 US Census [36]. There are: 29, 28, $95,39,41,124,27,16,12$, and 14 questions in anger/ anxiety, depression, fatigue, pain behavior, pain interference, physical function, sleep disturbance, sleep impairment, social impairment and social roles domains, respectively. The CAT presents a select group of questions out of the aforementioned domains (between 4 and 12 questions per domain) for patients to answer. Based on the responses to the first set of questions, the CAT continues to present more questions until the responses satisfy the preset reliability $(80 \%)$. There are five possible responses for each question (never, rarely, sometimes, often, and always) in the domains with the exception of six possible responses in the pain behavior domain (had no pain, never, rarely, sometimes, often, and always). The results of the questionnaire are presented as a $t$ score and a standard deviation based on the standardized US population. The mean $t$ score is 50 and the standard deviation is 10 . A typical printout of the PROMIS CAT is found in the supplementary material.

\section{Statistical Analysis}

Most data were presented as mean \pm standard deviation and median interquartile range (IQR) in months where it was appropriate. An unpaired $t$ test was used for comparisons of continuous variables and Chi-square or the Fisher exact tests were used for categorical variables. A nominal $p$ value of less than or equal to 0.05 was considered significant. Significant individual HRQOL domain and domain groups within PROMIS tools were derived using principal component analysis as described below. Cox proportional hazards regression modeling was used for significant domain groups for times to first hospitalization and re-hospitalization. Statistical analysis was performed with SPSS software for Windows, version 23 (SPSS, Inc, Chicago, IL).

\section{Significant Individual HRQOL Domains}

To reduce the number of variables in the analysis and to weigh individual PROMIS domains, an exploratory factor analysis with a principal component extraction method and varimax rotation was performed on the correlation matrix. Initially, using a minimum eigenvalue criteria of 1, two factors were identified that account for $68 \%$ of the variance. We wished to account for at least $70 \%$ of the variability, so we settled on a three-factor solution. The threefactor exploratory analysis showed three major domain groups which accounted for $77 \%$ of the variability. These were daily function-related (fatigue, physical function, social roles/activities, and sleep disturbance and impairment), mood-related (anxiety, anger, and depression), and pain-related (pain behavior/impact). Composite reliability indices calculated for the variables identified as contributing significantly to the HRQOL domains resulted in values of 0.884 for the daily function domain group, 0.867 for the mood domain group and 0.868 for the pain domain group.

\section{Outcome Measures}

Patients were followed prospectively at 3- to 6-month intervals at our outpatient clinics. During follow-up, all non-elective hospitalizations, death, and transplant were recorded. Subsequent hospitalizations after liver transplantation were not recorded. Non-elective hospitalizations were further categorized as liver related or liver unrelated (i.e., HE-related hospitalizations, renal ascites related, infections, portal hypertension related bleeding). Elective hospitalizations for procedures or studies were not included.

If a patient could not be seen at our outpatient clinics, they were tracked via our electronic medical system for any hospitalizations, death, and transplant with confirmation with telephone calls by the study staff. Time from initial PROMIS evaluation to first hospitalization and subsequent re-hospitalization(s) were recorded in months.

\section{Outcome Prediction Models}

Individual HRQOL domains were identified through factor analysis for each patient using the PROMIS domains as described above. Cox proportional hazards regression modeling was then utilized if any of the identified individual HRQOL domains had a significant impact of interest (i.e., time to first hospitalization and time to re-hospitalization). Backward elimination was performed and only individual HRQOL domains with a $p$ value less than 0.05 were retained. In addition, a univariate analysis was performed on known and potential variables for the outcomes of interest. These were age, gender, education, MELD score, serum sodium, albumin, ammonia, and complications of cirrhosis (history of $\mathrm{HE}$, ascites, and variceal bleeding). Variables that were significant on univariate analysis were then combined with the three individual domain groups and final models were constructed in a similar manner as described above (i.e., backward elimination with a significance level of 0.05 ).

\section{Results}

A total of 286 patients met enrollment criteria. All patients completed the PROMIS CAT at their first clinic visit. Baseline demographic characteristics of the study 
Table 1 Patient demographics

\begin{tabular}{|c|c|}
\hline & $N=286$ \\
\hline Age, mean \pm SD & $56.78 \pm 7.77$ \\
\hline Years of education, mean \pm SD & $13.50 \pm 2.37$ \\
\hline \multicolumn{2}{|l|}{ Etiology of cirrhosis, $n(\%)$} \\
\hline $\mathrm{HCV}$ & $108(38 \%)$ \\
\hline NASH & $69(24 \%)$ \\
\hline Alcoholic & $29(10 \%)$ \\
\hline Alcoholic and HCV & $25(9 \%)$ \\
\hline Other & $55(19 \%)$ \\
\hline Serum sodium $(\mathrm{mmol} / \mathrm{L})$ mean $\pm \mathrm{SD}$ & $138.00 \pm 7.65$ \\
\hline Serum albumin $(\mathrm{g} / \mathrm{dL})$ mean $\pm \mathrm{SD}$ & $3.44 \pm 0.71$ \\
\hline Serum ammonia $(\mu \mathrm{mol} / \mathrm{L})$ mean $\pm \mathrm{SD}$ & $52.67 \pm 30.53$ \\
\hline MELD score mean \pm SD & $12.70 \pm 5.80$ \\
\hline Ascites, $n(\%)$ & $104(36 \%)$ \\
\hline History of HE, $n(\%)$ & $113(40 \%)$ \\
\hline History of variceal bleeding, $n(\%)$ & $38(13 \%)$ \\
\hline Narcotic use, $n(\%)$ & $28(10 \%)$ \\
\hline NSAID use, $n(\%)$ & $28(10 \%)$ \\
\hline Anti-anxiolytics use, $n(\%)$ & $23(8 \%)$ \\
\hline SSRI/SSNRI use, $n(\%)$ & $45(16 \%)$ \\
\hline Other anti-depressants use, $n(\%)$ & $22(8 \%)$ \\
\hline \multicolumn{2}{|l|}{ PROMIS CAT tools, mean $\pm S D$} \\
\hline \multicolumn{2}{|l|}{ Mood domain group } \\
\hline Anger & $51.13 \pm 8.27$ \\
\hline Anxiety & $53.87 \pm 7.38^{\dagger}$ \\
\hline Depression & $51.22 \pm 8.64$ \\
\hline \multicolumn{2}{|l|}{ Daily function domain group } \\
\hline Fatigue & $55.38 \pm 9.38^{\dagger}$ \\
\hline Physical function* & $41.75 \pm 8.03^{\dagger}$ \\
\hline Sleep disturbance & $55.21 \pm 10.33$ \\
\hline Sleep-related impairment & $55.01 \pm 9.92^{\dagger}$ \\
\hline Discretionary social activities* & $45.88 \pm 7.98^{\dagger}$ \\
\hline Social roles* & $43.21 \pm 8.38^{\dagger}$ \\
\hline \multicolumn{2}{|l|}{ Pain domain group } \\
\hline Pain behavior & $53.16 \pm 9.63^{\dagger}$ \\
\hline Pain interference & $55.02 \pm 10.59$ \\
\hline
\end{tabular}

HE, hepatic encephalopathy; EVB, esophageal variceal bleed, M, male; F, female; MELD, Model for End-Stage Liver Disease; NSAID, nonsteroidal ant-inflammatory drug; SSRI, serotonin reuptake inhibitor; SNRI, serotonin-norepinephrine reuptake inhibitors; PROMIS, Patient-Reported Outcomes Measurement Information System; CAT, computerized adaptive measurement system

* A low score indicates worse symptoms; a high score indicates worse symptoms in the rest of the PROMIS scores

$\dagger$ Significantly different compared to PROMIS norms

population are shown in Table 1 . The majority was male $(67 \%)$, with a mean age of $56.8 \pm 7.8$ years, and had average years of education of $13.50 \pm 2.37$. The major etiologies of cirrhosis were hepatitis C (38\%) followed by nonalcoholic steatohepatitis (24\%) and alcoholic (10\%).
Patients were followed for a mean of 38 months [median IQR of 22-47]. During the follow-up period, 89 patients $(31 \%)$ were hospitalized [mean 12.5 months, median IQR (3-27)]) and 34 patients (12\%) were re-hospitalized [mean 10.5 months, median IQR $(3,28)]$. The most common reason for the first hospitalization was liver related $(23 \%)$ of which overt $\mathrm{HE}(9 \%)$, renal ascites related $(6 \%)$, and infections $(3 \%)$ were the most frequent etiologies. Similarly, the most common reason for re-hospitalization was liver-related (8\%) of which overt HE (3\%) and infections $(3 \%)$ were the most frequent etiologies. Fiftyseven patients died $(18 \%)$ and 73 patients were transplanted $(26 \%)$ during the follow-up period.

\section{PROMIS Analysis and Individual HRQOL Domains}

All PROMIS individual domains were significantly impaired compared to norms. The generation of domain groups from PROMIS tools is shown in Table 2. Of the domain groups identified, the daily function-related and mood/pain-related were significantly impaired compared to norms, $p<0.001$ and $<0.01$, respectively. Using multiple comparison corrections, there was no significant difference between PROMIS domains and use of narcotics, nonsteroidal ant-inflammatory drugs, serotonin reuptake inhibitors, serotonin-norepinephrine reuptake inhibitors, other anti-depressants, and anti-anxiolytics.

\section{Outcome Prediction}

Time to first hospitalization was predicted by HE [hazards ratio (HR) 1.5 (95\% CI 1.01-2.50, $p=0.04)$ ] and daily function group [HR $1.4(95 \%$ CI $1.1-1.8, p=0.01)$ ], independently. In contrast the pain group was predictive for time to re-hospitalization [(HR 1.6 (95\% CI: 1.10-2.30, $p=0.03)]$ as was HE [HR 2.1 (95\% CI 1.10-4.30, $p=0.03)]$ (Table 3).

\section{Discussion}

With the prolongation of survival in cirrhotic patients, addressing individual domains that drive HRQOL is important because it can quantify the impact of disease [35], define response to treatment [12], and potentially reduce costs by allocating resources to specific patient populations [35]. In our study, we identified two major domain groups within HRQOL, daily function and painrelated PROMIS measures, that predicted hospitalizations and re-hospitalizations independent of the MELD score and other markers of disease severity.

Confirming prior studies using PROMIS [30] and other tools [22-25], we found that majority of impairment was 
Table 2 Domain groups within PROMIS measures-factor loadings

\begin{tabular}{llcccc}
\hline Domain groups & PROMIS measures & Factor 1 & Factor 2 & Factor 3 & Final communality estimates \\
\hline Daily function & Social role & 87 & -11 & -24 & 0.8329 \\
& Social activity & 79 & -13 & -22 & 0.6837 \\
& Physical functioning & 78 & -3 & -40 & 0.7647 \\
& Fatigue & -66 & 38 & 31 & 0.6718 \\
& Sleep disturbance & -66 & 41 & 12 & 0.6169 \\
& Sleep-related impairment & -71 & 43 & 15 & 0.7140 \\
Mood & Anxiety & -17 & 85 & 25 & 0.8065 \\
& Anger & -14 & 83 & 4 & 0.7162 \\
Pain & Depression & -25 & 81 & 22 & 0.7590 \\
& Pain behavior & -30 & 27 & 88 & 0.9350 \\
\hline
\end{tabular}

The italic values indicate the domains that significantly impact that particular factor

* Overall, the variability in individual variables explained by the three factor solutions ranges from a low of approximately $62 \%$ for Sleep Disturbance to a maximum of approximately $94 \%$ for pain impact and pain behavior

HRQOL, health-related quality of life; PROMIS, Patient-Reported Outcomes Measurement Information System

Table 3 Predictors of first hospitalization and re-hospitalization

\begin{tabular}{|c|c|c|c|c|c|c|c|c|}
\hline \multirow[t]{3}{*}{ Variables } & \multicolumn{4}{|c|}{ First hospitalization } & \multicolumn{4}{|c|}{ Re-hospitalization } \\
\hline & \multicolumn{2}{|c|}{ Univariate } & \multicolumn{2}{|c|}{ Multivariate } & \multicolumn{2}{|c|}{ Univariate } & \multicolumn{2}{|c|}{ Multivariate } \\
\hline & $p$ value & HR $(95 \%$ CI $)$ & $p$ value & HR $(95 \%$ CI $)$ & $p$ value & HR $(95 \%$ CI $)$ & $p$ value & HR $(95 \%$ CI $)$ \\
\hline History of HE & 0.0007 & $2.07(1.36,3.12)$ & 0.04 & $1.57(1.02,2.45)$ & 0.007 & $2.58(1.30,5.11)$ & 0.03 & $2.14(1.06,4.33)$ \\
\hline Daily Function Group & 0.0015 & $0.68(0.53,0.86)$ & 0.017 & $1.37(1.06,1.77)$ & 0.061 & $0.68(0.46,1.02)$ & - & - \\
\hline Mood Group & 0.52 & $1.07(0.87,1.33)$ & - & - & 0.92 & $0.98(0.70,1.39)$ & - & - \\
\hline Pain Group & 0.15 & $1.17(0.94,1.46)$ & - & - & 0.0185 & $1.62(1.08,2.41)$ & 0.02 & $1.56(1.05,2.33)$ \\
\hline
\end{tabular}

MELD, Model for End-Stage Liver Disease; HE, hepatic encephalopathy; HR, hazard ratio; CI, confidence interval

related to the daily function groups. This is to be expected given the impairments in physical function and sleep that are prevalent in cirrhotic patients. Moreover, because of their decrement in physical function, patients may feel alienated in their community and family since they may perceive themselves as non-productive members of society. This can contribute to further impairment in social roles and activities. Frailty, which studies the physical function aspect of daily function-related domains, has already been associated with survival and re-hospitalizations [12]. The current study findings define the concomitant impact of physical function with social role and sleep issues on health-related outcomes. These findings point toward the importance of assessing daily function concerns at each clinical visit, both physical and mental, along with communicating the plan of care with patients and their family members. Examples of this would be addressing nutritional status to improve sarcopenia and muscle strength [36], and sleep disturbances which can be a sign of impending HE [37] or undiagnosed obstructive sleep apnea [38].
Translating subjective complaints into clinically relevant objective outcomes is always challenging. This is especially problematic in clinics where practitioners are often not trained to handle PROs, which are usually beyond their comfort zone. One example case would be in addressing pain in cirrhosis where escalating analgesic therapy is often complicated by concomitant clinical and pharmacological interactions. A multidisciplinary approach to pain is often lacking in most practices, which could explain the significant impairment in the PROMIS pain scores in our cohort. This is particularly important given that up to $77 \%$ of cirrhotics experience moderate-to-severe pain constantly [39], which is comparable to those with lung cancer and colon cancer [40]. This can eventually lead to mismanagement of pain medications or overtreatment, increase in healthcare utilization [48], and in the current study, re-hospitalizations. This was supported by a recent study in which opioid use was associated with re-hospitalizations in cirrhotics recruited as inpatients [41]. Thus, it is of high importance to incorporate evidence-based pain 
management strategies, such as the Centers of Disease Control and Prevention guideline for prescribing opioids for chronic pain [42], and encourage a multidisciplinary approach with access to hospice and palliative care medicine [43]. Lastly, while our study did not find any association with mood and hospitalizations/re-hospitalizations, these conditions are nevertheless important from a daily function standpoint and in other studies to liver-related mortality [44].

To our knowledge, this is the first study to identify major individual domain groups within a HRQOL measure and its impact on hospitalizations. However, there were few limitations of the study. The first was a lack of a separate validation cohort to substantiate our findings, and thus further validation is required to build on the current results. Second, we did not use death and or transplant as a composite outcome since the focus of study was hospitalizations. Lastly, we were unable to control for the number of medications on discharge given its possible impact on re-hospitalizations. Future work should study how treatments affect these individual HRQOL domains and if dedicated treatment reduces hospitalizations. Another avenue for further research would be to evaluate the effectiveness of these domains, along with our current objective measures for determining liver transplant eligibility and prognosis after transplant.

We conclude that daily function-related and pain-related domain groups within PROMIS tools can predict hospitalizations and re-hospitalizations independent of objective clinical measures in cirrhotic outpatients. Therefore, efforts to incorporate PROs and their individual domains into clinical practice could improve prognostication and help in focused treatments.

Acknowledgments This study was partly supported by the NIDDK RO1DK087013 and VA Merit Review Grant CX10076 awarded to Jasmohan S. Bajaj.

Author's contributions JSB contributed to study concept and design. LRT, JSB, and KRP analyzed the data. Manuscript preparation was carried out by KRP, JSB, and LRT. JSB, RKS, AJS, RTS, SM, MS, VL, MF, PP, MBW, AF, JBW, and EAG recruited the patients and conducted the study. Manuscript was reviewed by all the authors.

\section{Compliance with ethical standards}

Conflict of interest None.

\section{References}

1. GBD 2013 Mortality and Causes of Death Collaborators. Global, regional, and national age-sex specific all-cause and causespecific mortality for 240 causes of death, 1990-2013: a systematic analysis for the global burden of disease study 2013.
Lancet. 2015;385:117-171. doi:10.1016/S0140-6736(14)61682-

2. Younossi ZM, Koenig AB, Abdelatif D, Fazel Y, Henry L, Wymer M. Global epidemiology of nonalcoholic fatty liver disease-meta-analytic assessment of prevalence, incidence, and outcomes. Hepatology. 2016;64:73-84. doi:10.1002/hep.28431.

3. Udompap P, Kim D, Kim WR. Current and future burden of chronic nonmalignant liver disease. Clin Gastroenterol Hepatol. 2015;13:2031-2041. doi:10.1016/j.cgh.2015.08.015.

4. Tapper EB, Finkelstein D, Mittleman MA, Piatkowski G, Chang M, Lai M. A quality improvement initiative reduces 30-day rate of readmission for patients with cirrhosis. Clin Gastroenterol Hepatol. 2016;14:753-759. doi:10.1016/j.cgh.2015.08.041.

5. Tapper EB, Halbert B, Mellinger J. Rates of and reasons for hospital readmissions in patients with cirrhosis: a multistate population-based cohort study. Clin Gastroenterol Hepatol. 2016;14:1181-1188.e2. doi:10.1016/j.cgh.2016.04.009.

6. Bajaj JS, Reddy KR, Tandon P, et al. The 3-month readmission rate remains unacceptably high in a large north american cohort of patients with cirrhosis. Hepatology. 2016;64:200-208. doi:10. 1002/hep.28414.

7. Kanwal F, Hays RD, Kilbourne AM, Dulai GS, Gralnek IM. Are physician-derived disease severity indices associated with healthrelated quality of life in patients with end-stage liver disease? Am J Gastroenterol. 2004;99:1726-1732. doi:10.1111/j.1572-0241. 2004.30300.x.

8. Volk ML, Tocco RS, Bazick J, Rakoski MO, Lok AS. Hospital readmissions among patients with decompensated cirrhosis. Am J Gastroenterol. 2012;107:247-252. doi:10.1038/ajg.2011.314.

9. Berman K, Tandra S, Forssell K, et al. Incidence and predictors of 30-day readmission among patients hospitalized for advanced liver disease. Clin Gastroenterol Hepatol. 2011;9:254-259. doi:10.1016/j.cgh.2010.10.035.

10. Ganesh S, Rogal SS, Yadav D, Humar A, Behari J. Risk factors for frequent readmissions and barriers to transplantation in patients with cirrhosis. PLoS One. 2013;8:e55140. doi:10.1371/ journal.pone.0055140.

11. Singal AG, Rahimi RS, Clark C, et al. An automated model using electronic medical record data identifies patients with cirrhosis at high risk for readmission. Clin Gastroenterol Hepatol. 2013;11:1335-1341.e1. doi:10.1016/j.cgh.2013.03.022.

12. Tapper EB, Finkelstein D, Mittleman MA, Piatkowski G, Lai M. Standard assessments of frailty are validated predictors of mortality in hospitalized patients with cirrhosis. Hepatology. 2015;62:584-590. doi:10.1002/hep.27830.

13. Marchesini G, Bianchi G, Amodio P, et al. Factors associated with poor health-related quality of life of patients with cirrhosis. Gastroenterology. 2001;120:170-178.

14. Martin LM, Sheridan MJ, Younossi ZM. The impact of liver disease on health-related quality of life: a review of the literature. Curr Gastroenterol Rep. 2002;4:79-83.

15. Orr JG, Homer T, Ternent L, et al. Health related quality of life in people with advanced chronic liver disease. J Hepatol. 2014;61:1158-1165. doi:10.1016/j.jhep.2014.06.034.

16. Kanwal F, Gralnek IM, Hays RD, et al. Health-related quality of life predicts mortality in patients with advanced chronic liver disease. Clin Gastroenterol Hepatol. 2009;7:793-799. doi:10. 1016/j.cgh.2009.03.013.

17. Bajaj JS, Wade JB, Gibson DP, et al. The multi-dimensional burden of cirrhosis and hepatic encephalopathy on patients and caregivers. Am J Gastroenterol. 2011;106:1646-1653. doi:10. 1038/ajg.2011.157.

18. Bajaj JS, White MB, Unser AB, et al. Cirrhotic patients have good insight into their daily functional impairment despite prior hepatic encephalopathy: comparison with PROMIS norms. Metab Brain Dis. 2016. doi:10.1007/s11011-016-9860-8. 
19. Sanyal A, Younossi ZM, Bass NM, et al. Randomised clinical trial: rifaximin improves health-related quality of life in cirrhotic patients with hepatic encephalopathy-a double-blind placebocontrolled study. Aliment Pharmacol Ther. 2011;34:853-861. doi:10.1111/j.1365-2036.2011.04808.x.

20. Younossi ZM, Guyatt G, Kiwi M, Boparai N, King D. Development of a disease specific questionnaire to measure health related quality of life in patients with chronic liver disease. Gut. 1999;45:295-300.

21. Gralnek IM, Hays RD, Kilbourne A, et al. Development and evaluation of the liver disease quality of life instrument in persons with advanced, chronic liver disease-the LDQOL 1.0. Am J Gastroenterol. 2000;95:3552-3565.

22. Kanwal F, Spiegel BM, Hays RD, et al. Prospective validation of the short form liver disease quality of life instrument. Aliment Pharmacol Ther. 2008;28:1088-1101. doi:10.1111/j.1365-2036. 2008.03817.x.

23. van der Plas SM, Hansen BE, de Boer JB, et al. The liver disease symptom index 2.0; validation of a disease-specific questionnaire. Qual Life Res. 2004;13:1469-1481.

24. Tsai LH, Lin CM, Chiang SC, Chen CL, Lan SJ, See LC. Symptoms and distress among patients with liver cirrhosis but without hepatocellular carcinoma in taiwan. Gastroenterol Nurs. 2014;37:49-59. doi:10.1097/SGA.0000000000000020.

25. Fritz E, Hammer J. Gastrointestinal symptoms in patients with liver cirrhosis are linked to impaired quality of life and psychological distress. Eur J Gastroenterol Hepatol. 2009;21: 460-465.

26. Nardelli S, Pentassuglio I, Pasquale C, et al. Depression, anxiety and alexithymia symptoms are major determinants of health related quality of life (HRQoL) in cirrhotic patients. Metab Brain Dis. 2013;28:239-243. doi:10.1007/s11011-012-9364-0.

27. Rothrock NE, Hays RD, Spritzer K, Yount SE, Riley W, Cella D. Relative to the general US population, chronic diseases are associated with poorer health-related quality of life as measured by the patient-reported outcomes measurement information system (PROMIS). J Clin Epidemiol. 2010;63:1195-1204. doi:10. 1016/j.jclinepi.2010.04.012.

28. Cella D, Riley W, Stone A, et al. The patient-reported outcomes measurement information system (PROMIS) developed and tested its first wave of adult self-reported health outcome item banks: 2005-2008. J Clin Epidemiol. 2010;63:1179-1194. doi:10.1016/j.jclinepi.2010.04.011.

29. Liu H, Cella D, Gershon R, et al. Representativeness of the patient-reported outcomes measurement information system internet panel. J Clin Epidemiol. 2010;63:1169-1178. doi:10. 1016/j.jclinepi.2009.11.021.

30. Bajaj JS, Thacker LR, Wade JB, et al. PROMIS computerised adaptive tests are dynamic instruments to measure health-related quality of life in patients with cirrhosis. Aliment Pharmacol Ther. 2011;34:1123-1132. doi:10.1111/j.1365-2036.2011.04842.x.

31. Ahluwalia V, Wade JB, White MB, et al. Liver transplantation significantly improves global functioning and cerebral processing. Liver Transpl. 2016;22:1379-1390. doi:10.1002/lt.24498.

32. Mar J, Larranaga I, Arrospide A, Begiristain JM. Impact of disability on different domains of health-related quality of life in the noninstitutionalized general population. Clinicoecon Outcomes Res. 2010;2:97-103.

33. Quinten C, Martinelli F, Coens C, et al. A global analysis of multitrial data investigating quality of life and symptoms as prognostic factors for survival in different tumor sites. Cancer. 2014;120:302-311. doi:10.1002/cncr.28382.

34. Saarni SI, Suvisaari J, Sintonen H, et al. Impact of psychiatric disorders on health-related quality of life: general population survey. Br J Psychiatry. 2007;190:326-332.

35. Neff GW, Duncan CW, Schiff ER. The current economic burden of cirrhosis. Gastroenterol Hepatol (N Y). 2011;7:661-671.

36. Thandassery RB, Montano-Loza AJ. Role of nutrition and muscle in cirrhosis. Curr Treat Options Gastroenterol. 2016;14:257-273. doi:10.1007/s11938-016-0093-z.

37. Montagnese S, Turco M, Amodio P. Hepatic encephalopathy and sleepiness: an interesting connection? J Clin Exp Hepatol. 2015;5:S49-S53. doi:10.1016/j.jceh.2014.06.006.

38. Bajaj JS, Thacker LR, Leszczyszyn D, et al. Effects of obstructive sleep apnea on sleep quality, cognition, and driving performance in patients with cirrhosis. Clin Gastroenterol Hepatol. 2015;13:390-397.e1. doi:10.1016/j.cgh.2014.08.028.

39. Madan A, Barth KS, Balliet WE, et al. Chronic pain among liver transplant candidates. Prog Transplant. 2012;22:379-384.

40. Roth K, Lynn J, Zhong Z, Borum M, Dawson NV. Dying with end stage liver disease with cirrhosis: insights from SUPPORT. study to understand prognoses and preferences for outcomes and risks of treatment. J Am Geriatr Soc. 2000;48:S122-S130.

41. Acharya C, Betrapally NS, Gillevet PM, et al. Chronic opioid use is associated with altered gut microbiota and predicts readmissions in patients with cirrhosis. Aliment Pharmacol Ther. 2017;45:319-331. doi:10.1111/apt.13858.

42. Dowell D, Haegerich TM, Chou R. CDC guideline for prescribing opioids for chronic pain-United States, 2016. MMWR Recomm Rep. 2016;65:1-49. doi:10.15585/mmwr.rr6501e1.

43. Potosek J, Curry M, Buss M, Chittenden E. Integration of palliative care in end-stage liver disease and liver transplantation. $J$ Palliat Med. 2014;17:1271-1277. doi:10.1089/jpm.2013.0167.

44. Russ TC, Kivimaki M, Morling JR, Starr JM, Stamatakis E, Batty GD. Association between psychological distress and liver disease mortality: a meta-analysis of individual study participants. Gastroenterology. 2015;148:958-966.e4. doi:10.1053/j.gastro.2015. 02.004 . 